\title{
INTERFACE EFFECTS IN THE MODEL OF $\delta$-POTENTIAL FOR 2D SEMICONDUCTOR QUANTUM STRUCTURES
}

\author{
Yu.G. SEMENov \\ Institute of Semiconductor Physics, National Academy of Sciences of Ukraine \\ Prospect Nauki 45, Kiev 252650, Ukraine
}

Here we show that the enhancement of exchange field at the nonmagnetic-semimagnetic semiconductor interface can be described by $\delta$-localized exchange field. In this model, the wave functions of electron confined in quantum well with semimagnetic barriers can be found analytically for an arbitrary orientation of magnetic field $B$ with respect to growth axis $C$. Two cases corresponding to $\boldsymbol{B} \| \boldsymbol{C}$ and $\boldsymbol{B} \perp \boldsymbol{C}$ are considered. The comparison of present approach with experimental data shows that the proposed method is an efficient tool for interface investigation in $2 \mathrm{D}$ quantum structures.

PACS numbers: 73.20.Dx, 78.66.-w

\section{Introduction}

Common approach to quantitative description of carrier confinement in quantum well (QW) consists in approximation of magnetic ions distribution by rectangular profile $x(z)=x \theta\left(|z|-L_{\mathrm{w}} / 2\right)$, where $L_{\mathrm{w}}$ is a width of the QW with growth axis $C \| O Z ; \theta(z)$ being the Heaviside step function. The similar profile would be expected for both electron and hole band-offset potentials. Contribution of exchange interaction induced by external magnetic field does not generate the same kind of potential profile due to the special conditions in the interface of $\mathrm{Mn}$ layers [1-3]. Detailed analysis of interface exchange potential and its influence on the exciton energy spectra in $\mathrm{Cd}_{1-x} \mathrm{Mn}_{x} \mathrm{Te} / \mathrm{CdTe} / \mathrm{Cd}_{1-x} \mathrm{Mn}_{x}$ Te quantum structure in magnetic field was done in [4].

Here we propose another description of the interface magnetic potential based on the assumption of large difference between the interface potential scale $\Delta l$ and QW width $L_{\mathrm{w}}: \Delta l \ll L_{\mathrm{w}}$. In this case the interface potential is reduced to the $\delta$-function with coefficient $A$ equal to the integral of the interface potential $U_{\text {if }}(z)$. 


\section{Theoretical outline}

Let us represent the heterostructure potential by the sum of rectangular potential and interface one $U_{\text {if }}(z)$ localized at the length scale $\Delta l$ in the vicinity of a potential step. The contribution of $U_{\text {if }}(z)$ to the electron (hole) potential energy is $E_{\text {if }}=\left\langle\psi\left|U_{\text {if }}(z)\right| \psi\right\rangle$ where $\psi(z)$ is an exact wave function. If the typical scale $L_{\mathrm{w}}$ of the $\psi(z)$ oscillations is larger than $\Delta l$, the $E_{\mathrm{if}}$ can be approximated by $E_{\mathrm{if}}^{(0)}=A\left|\psi\left(L_{\mathrm{w}} / 2\right)\right|^{2}$. Thus, the $E_{\mathrm{if}}^{(0)}$ corresponds to contribution of the interface Hamiltonian

$$
H_{\mathrm{if}}^{(0)}=A \delta\left(z-L_{\mathrm{w}} / 2\right) \text {. }
$$

The factor $A=\int U_{\mathrm{if}}(z) \mathrm{d} z$ depends on the specific distribution of magnetic ions and should be considered as a fitting parameter.

Taking into account that the exchange potential is proportional to local magnetization which is distributed over wide range of values [4], the magnetic field dependence of parameter $A$ is assumed to be well approximated by the modified Brillouin function with the effective parameters $T_{\text {if }}$ and $S_{\text {if }}$ being similar to the parameters $T_{0}$ and $S_{0}$ used for bulk DMS

$$
A \propto S_{\text {if }} B_{S}\left(\frac{g \mu_{\mathrm{B}} B S}{k_{\mathrm{B}}\left(T+T_{\mathrm{if}}\right)}\right) .
$$

Thus the factors $A$ can be taken in the form

$$
\begin{array}{ll}
A_{\mathrm{e}}=\Delta l G_{\mathrm{e}}^{\mathrm{if}} S_{\mathrm{e}}, & G_{\mathrm{e}}^{\mathrm{if}}=N_{0} \alpha x\left(S_{\mathrm{if}} / S\right) S B_{S}(y), \\
A_{\mathrm{h}}=\Delta l G_{\mathrm{h}}^{\mathrm{if}} S_{\mathrm{h}}, & G_{\mathrm{h}}^{\text {if }}=N_{0} \beta x\left(S_{\mathrm{if}} / S\right) S B_{S}(y),
\end{array}
$$

for electron and hole, respectively, with $G_{\mathrm{e}(\mathrm{h})}^{\mathrm{if}}$ directed along external magnetic field, and $y=g \mu_{\mathrm{B}} B S / k_{\mathrm{B}}\left(T+T_{\mathrm{if}}\right)$. The electron and hole exchange potentials generate two free parameters only, the $T_{\mathrm{if}}$ and a product $\Delta L=\Delta l\left(S_{\mathrm{if}} / S\right)$.

\section{The GSS of excitonic spectra in DMS QWs at the $B \| C$}

Let us apply the proposed model to description of the giant spin splitting (GSS) of reflectivity excitonic spectra for the $\mathrm{Cd}_{1-x} \mathrm{Mn}_{x} \mathrm{Te} / \mathrm{CdTe} / \mathrm{Cd}_{1-x} \mathrm{Mn}_{x} \mathrm{Te}$ structure. For simplicity the consideration will be restricted to electron and hole ground states.

The Schrödinger equation and the conditions of continuity of $\psi$-function and discontinuity of its derivative at the interface describe fully the problem under consideration. The $\delta$-potential power is represented in derivative discontinuity value $\sigma \Delta l G_{\mathrm{e}(\mathrm{h})}^{\mathrm{if}} / L_{\mathrm{w}}^{2} E_{0} \psi\left(L_{\mathrm{w}} / 2\right)$ which is the difference of $\partial \psi / \partial z$ calculated at barrier and QW near the interface; $\sigma= \pm 1 / 2, E_{0}=\hbar^{2} / 2 m^{*} L_{\mathrm{w}}^{2}, m^{*}$ is an effective mass of electron (hole). The confinement energy $E$ can be found from equation

$$
\sqrt{\varepsilon} \tan \frac{\sqrt{\varepsilon}}{2}=\sqrt{u_{\mathrm{e}(\mathrm{h})}+g_{\mathrm{e}(\mathrm{h})} \sigma-\varepsilon}+g_{\mathrm{e}(\mathrm{h})}^{\mathrm{if}} \sigma,
$$

where $\varepsilon=E / E_{0}, u_{\mathrm{e}(\mathrm{h})}=U_{\mathrm{e}(\mathrm{h})} / E_{0}, g_{\mathrm{e}(\mathrm{h})}=G_{\mathrm{e}(\mathrm{h})} / E_{0}$ are taken in $E_{0}$ units and $g_{\mathrm{e}(\mathrm{h})}^{\mathrm{if}}=\left(\Delta l / L_{\mathrm{w}}\right) G_{\mathrm{e}(\mathrm{h})}^{\mathrm{if}} / E_{0}$. Equation (3) reduces to the well-known one for rectangular potential in the limit $g_{\mathrm{e}(\mathrm{h})}^{\mathrm{if}} \rightarrow 0$. 
The electron-hole transition energy is affected also by the electron-hole Coulomb interaction and the strain effect [4]. To avoid consideration of these effects, the analysis is restricted to the GSS of ground states only. Thus the procedure of excitonic GSS calculation consists of calculation of the electron and hole spin splitting by the magnetic field with the aid of Eq. (3) and summing over all pairs of electron and hole energy branches corresponding to some optical polarization.
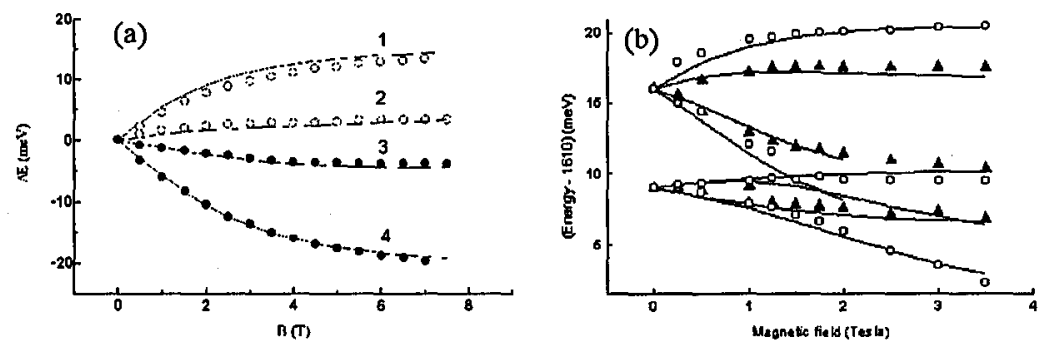

Fig. 1. Comparison of calculated and measured GSS for excitonic spectra in two different orientations of magnetic field $\boldsymbol{B}:$ (a) $\boldsymbol{B} \| \boldsymbol{C}$, (b) $\boldsymbol{B} \perp \boldsymbol{C}$. Symbols are the experiments of Ref. [5] (a) and Ref. [7] (b): circles and triangles correspond to $\sigma$ - and $\pi$-polarizations.

Figure 1a shows the comparison of calculated and measured GSS spectra for $L_{\mathrm{w}}=88 \AA, x=0.22$ (curves 2 and 3 ), and $L_{\mathrm{w}}=30 \AA, x=0.18$ (curves 1 and 4 ). The coincidence of theory with the experimental data [5] is good if one chooses the parameters $\Delta L=2.8 \AA, T_{\mathrm{if}}=2 \mathrm{~K}$ and $\Delta L=1.25 \AA, T_{\mathrm{if}}=2 \mathrm{~K}$ respectively. Besides, diamagnetic shift was taken into account and valence band-offset parameters $Q=0.4$ was chosen according to [6].

It can be possible to realise confinement states caused by interface attractive potential exceeding QW contribution. This situation was considered for the case of QW structure [5] with $L_{\mathrm{w}}=88 \AA$, but with enhanced interface parameter

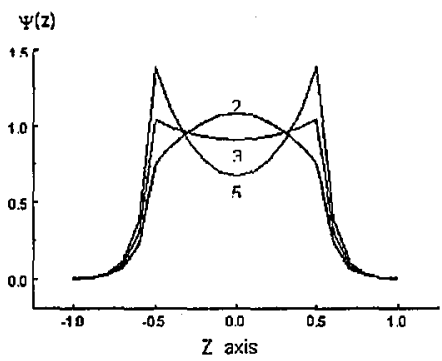

Fig. 2. Transformation of $z$-component shape of $\psi$ function while a magnetic field passes through the critical value $B_{\mathrm{c}} \approx 2.7 \mathrm{~T}$. Calculation was performed for $x=0.22$ and $\Delta L=5 \AA$. The numbers near the curves indicate the strength of magnetic field in T. The QW width $L_{z}=88 \AA$ is used for unit of $z$-axis. 
$\Delta L=5 \AA$. Heavy hole states are affected by transition to negative energy with respect to bottom of QW potential near $B_{\mathrm{c}}=2.7 \mathrm{~T}$. The shape of $\psi$-function calculated for different magnetic fields is shown in Fig. 2. Evidently the transition to the case of $E<0$ can be revealed in the reduction of excitonic line intensity for $B>B_{\mathrm{c}}$.

\section{The GSS of excitonic spectra at the $B \perp C$}

This case does not require a special consideration for electron states. As to hole states, the spin-orbit interaction does not commute now with the operator of exchange splitting which leads to heavy hole-light hole states mixing. This problem was considered earlier in several papers [7-9] but the interface potential was not taken into account.

According to $[7,8]$ the $O Z$ axis is directed along $\boldsymbol{B}$, while growth direction $C$ should be designated as $O X$ axis. Under these parametrizations the Luttinger equation (LE) can be reduced to the two mutually independent twofold systems of the equations in the basis set $|3 / 2\rangle,|-1 / 2\rangle$ and $|-3 / 2\rangle,|1 / 2\rangle$. The hole energy spectrum is determined by the solution of characteristic equation for the system of linear equations for eight components of $\psi$-function. They consist of the LEs for barrier and QW and the conditions of continuity of $\psi$-function and discontinuity of its derivative at the $\mathrm{QW}$ and barrier interface. The power of interface $\delta$-potential appears now in the discontinuity boundary condition for the $\psi$-function

$$
\lim _{\xi \rightarrow 1 / 2-0} \widehat{V} \psi=\lim _{\xi \rightarrow 1 / 2+0}(\widehat{V}+\widehat{D}) \psi,
$$

where

$$
\widehat{V}=\left(\begin{array}{cc}
-\left(\gamma_{1}+\gamma_{2}\right) \frac{\partial}{\partial \xi} & \sqrt{3} \gamma_{2} \frac{\partial}{\partial \xi} \\
\sqrt{3} \gamma_{2} \frac{\partial}{\partial \xi} & -\left(\gamma_{1}-\gamma_{2}\right) \frac{\partial}{\partial \xi}
\end{array}\right), \quad \widehat{D}=\left(\begin{array}{cc}
\frac{1}{2} g_{\text {if }} & 0 \\
0 & -\frac{1}{6} g_{\text {if }}
\end{array}\right) .
$$

Since $\gamma_{1}$ and $\gamma_{2}$ are the Luttinger parameters, the energy unit $E_{0}$ corresponds to free electron mass, $\xi=x / L_{\mathrm{w}}$. Two solutions of corresponding characteristic equation give the energy spectrum which represents one half of the matrix of the Schrödinger equation in basis $|3 / 2\rangle,|-1 / 2\rangle$. Other equation obtained in basis $|-3 / 2\rangle,|1 / 2\rangle$ can be formally deduced from previous one by changing $\boldsymbol{B}$ with $-\boldsymbol{B}$. In this way can be obtained the full spectrum representing the heavy hole and light hole splitting in QW in a magnetic field.

The numerical procedure for calculation of the energy spectrum and its application to the case of electron-hole optical transition has been realized. It was applied to the case of $\mathrm{Cd}_{1-x} \mathrm{Mn}_{x} \mathrm{Te} / \mathrm{CdTe} / \mathrm{Cd}_{1-x} \mathrm{Mn}_{x} \mathrm{Te}$ quantum structure with $L_{\mathrm{w}}=75 \AA, x=0.05$ [7]. Figure 1b shows the comparison of these calculations with experimental data presented in [7]. The free parameters chosen to best fitting are following: $\Delta L=1.5 \AA, T_{\text {if }}=0.5 \mathrm{~K}$. It can be shown that taking into account the interface enhancement of exchange field permits to get the better description of experimental data.

\section{Conclusion}

It has been shown that enhancement (or suppression) of interface potential energy caused by the specific magnetic properties of boundary magnetic ions 
in quantum structures has simple description in terms of the $\delta$-localized exchange field. This approximation permits to apply analytical approach to electron and hole wave functions for both Faraday and Voigt configurations. Detailed comparison with experiments done for different $\mathrm{Cd}_{1-x} \mathrm{Mn}_{x} \mathrm{Te} / \mathrm{CdTe} / \mathrm{Cd}_{1-x} \mathrm{Mn}_{x}$ Te structures lends support to the validity of the developed theory. The quantitative description of the complex picture of heavy hole-light hole excitonic splitting as well as light hole GSS needs to incorporate the stress effect as well as Coulomb and exchange electron-hole interaction into the model. Obviously, the developed method has many other applications for different physical phenomena in DMS quantum structures.

\section{Acknowledgment}

Author is grateful to S.M. Ryabchenko, D. Scalbert and V. Stephanovich for fruitful discussion of the questions raised in this paper. The present work was partially supported by grant No. INTAS-93-3658p and grant of the Ukrainian State Fund for Fundamental Research No. 2.4/638.

\section{References}

[1] See, for example, C. Benoit à la Guillaume, in: II-VI Compounds and Semimagnetic Semiconductors, Linz 1994, Eds. H. Heinrich, J.B. Mullin, Trans Tech, Zurich 1995, p. 549.

[2] J.A. Gaj, P. Kossacki, Nguyen The Khoi, in: Semiconductor Heteroepitaxy, Eds. B. Gil, R.-L. Aulombard, World Scientific, Singapore 1995, p. 202.

[3] W. Ossau, B. Kuhn-Heinrich, Physica B 184, 422 (1993).

[4] J.A. Gaj, W. Grieshaber, C. Boden-Deshayes, J. Cibert, G. Feuillert, Y. Merle d'Aubigné, A. Wasiela, Phys. Rev. B 50, 5512 (1994).

[5] B. Kuhn-Heinrich, M. Popp, W. Ossau, E. Bangert, G. Landwehr, Semicond. Sci. Technol. 8, 1239 (1993).

[6] M. Kutrowski, T. Wojtowicz, G. Cywinski, G. Karczewski, E. Janik, E. Dynowska, J. Kossut, P. Kossacki, R. Fiederling, A. Pfeuffer-Jeschke, W. Ossau, Acta Phys. Pol. A 90, 977 (1996).

[7] P. Peyla, A. Wasiela, Y. Merle d'Aubigné, D.E. Ashenford, B. Lunn, Phys. Rev. $B$ 47, 3783 (1993).

[8] B. Kuhn-Heinrich, W. Ossau, E. Bangert, A. Waag, G. Landwehr, Solid State Commun. 91, 413 (1994).

[9] B. Kuhn-Heinrich, W. Ossau, Mater. Sci. Forum 182-184, 491 (1995). 